\title{
Un musicien chez les coupeurs de têtes de Michel DINTRICH
}

\section{Gilles Bounoure}

\section{(2) OpenEdition Journals}

Édition électronique

URL : http://journals.openedition.org/jso/5979

DOI : $10.4000 /$ jso.5979

ISSN : 1760-7256

Éditeur

Société des océanistes

Édition imprimée

Date de publication : 15 décembre 2009

Pagination : 335-337

ISBN : 978-2-85430-026-0

ISSN : 0300-953x

\section{Référence électronique}

Gilles Bounoure, "Un musicien chez les coupeurs de têtes de Michel Dintrich », Journal de la Société des Océanistes [En ligne], 129 | juillet-décembre 2009, mis en ligne le 15 décembre 2009, consulté le 24 septembre 2020. URL : http://journals.openedition.org/jso/5979; DOI : https://doi.org/10.4000/jso. 5979

(c) Tous droits réservés 
ancestrales » (p. 255), et dans lequel l'imaginaire est constamment mis en relation avec l'historicité.

La revue thématique critique opérée par Sylvie Poirier sur des concepts ressassés en Australie n'a rien de scolaire et cette réévaluation a toute son utilité d'autant qu'elle a été réalisée avec finesse et, comme on l'a dit, avec une remarquable empathie. L'auteure a le don de présenter sur un mode narratif des notions qui ressortissent à des ontologies et des métaphysiques bien éloignées de celles de la tradition occidentale. Son récit - parce que son livre peut être considéré comme un long récit - traite de thèmes qui apparaissent au premier abord comme des thèmes abstraits, mais qui prennent, sous son analyse narrative, une épaisseur historique et quotidienne telle qu'ils deviennent rapidement des morceaux de vécu intense qui semblent nous incorporer dans l'analyse. Bref, une fois installés, on a envie de rester, tant la mise en « relation » avec les porteurs de rêves et de science du rêve finit par nous attacher à une relation où chacun trouve sa place.

S'il y avait une réserve à formuler, ce serait peut-être l'insatisfaction que l'on pourrait éprouver par rapport à un certain de nombre de termes endogènes manquants, même si une trentaine d'entre eux a été soigneusement épluchée au fil de l'écriture critique qui les a mis en scène. On regrettera aussi que les quelque quarante récits mobilisés pour servir l'argumentaire général de l'ouvrage ne soient pas transcrits dans la langue des Kukatja, ne serait-ce que pour contribuer à la patrimonialisation des « langues en danger ». On a beau dire que les langues sont dorénavant l'affaire des linguistes et qu'il faut respecter l'interdit de la mention des noms de personnes décédées, toute entreprise anthropologique majeure doit faire des corpus endogènes une priorité incontournable. Mais l'immersion dans la longue durée de la culture étudiée a sans aucun doute qualifié Sylvie Poirier pour faire parler autant les rêves que la science locale, qui trouve précisément dans les rêves non seulement une modalité d'expression, mais une de ses principales ressources. Aller jusqu'au bout de la ressource est l'itinéraire principal de l'ouvrage, indépendamment des kilomètres parcourus sur le terrain.

Raymond MAYER, Université Omar Bongo de Libreville et Université de Lyon 2

DinTRICH Michel, 2009. Un musicien chez les coupeurs de têtes, Paris, Mille et une nuits, 272 p., cahier de 16 p. d'illustrations couleur, nombreuses ill. noir et blanc dans le texte.

Entre les derniers jours de juin et les premiers d'août 1985, l'auteur de ce livre, un quinquagénaire français, guitariste classique de renom, attiré par les arts océaniens et les joies du dépaysement, visite le sud de l'Indonésie, en tâchant de rejoindre à moindres frais la mer d'Arafura et la côte des Casuarines, but de son voyage. C'est là qu'il espère recueillir des impressions... et des objets d'art asmat. Ancien élève d'une académie de peinture bien connue à Paris, il se remet pour l'occa- sion au dessin et à l'aquarelle. Selon le récit qu'il livre ici de son périple, les esquisses qu'il accumule dans son carnet (et dont quelques-unes sont reprises dans ce volume) lui seront parfois plus utiles auprès de ses interlocuteurs de rencontre que le vocabulaire malais ou les dollars dont il dispose - sans parler d'un appareil photographique dont son récit ne dit mot, mais d'où proviennent d'autres illustrations de son livre. S'y trouvent aussi reproduits certains des objets qu'il a rapportés en France, une série d'ornements de nez bipane, un mât mbis compatible avec l'ordinaire hauteur sous plafond d'un appartement parisien, ainsi qu'une photographie d'André Breton dans son atelier.

L'engouement de Michel Dintrich pour les arts asmat lui est venu, écrit-il, d'un bouclier de ce style qu'il a vu accroché au centre du grand mur de l'atelier du 42, rue Fontaine à Paris, plusieurs lustres après la mort du poète. Cet objet, aujourd'hui visible au centre Pompidou, mais absent des dernières photographies prises in situ du vivant de Breton, telles celles de Sabine Weiss en 1960, n'était pas la plus spectaculaire des pièces représentatives des arts du Pacifique accumulées dans cet atelier. Un crâne asmat, non cité par l'auteur, aurait pu tout aussi bien justifier sa prédilection exclusive et son projet de voyage, dont les ressorts demeurent ainsi obscurs ou confus. Maintes inexactitudes de fait (sur la date de la mort de Breton, p. 32, sur le nombre d'objets de la baie de Geelvink en sa possession, nettement supérieur à «deux», p. 30, comme le montre encore le film tourné en 1994 dans l'atelier par Frédéric Maze pour le centre Pompidou, etc.) suggèrent que l'auteur n'a pas été si attentif qu'il le dit à cette collection ou au contexte social qu'il lui prête, certaines des scènes qu'il rapporte en contrepoint de son récit ayant trouvé des témoins pour les contester. La part du livre consacrée au séjour chez les Asmat (pp. 140-225), abondamment ponctuée d'anecdotes complaisantes sur le passé scout, les premières armes ou les concerts du musicien, marque un intérêt non moins expéditif et superficiel à « ces guerriers redoutables, jadis réputés dans toute la NouvelleGuinée (sic) pour leur cruauté » (p. 145).

L'auteur en convient et semble s'en accommoder, il lui est « difficile de se débarrasser des archétypes véhiculés à propos des sauvages, surtout quand ces derniers sont réputés extrêmement dangereux, de surcroît anthropophages » (p. 168), car une fois que « ces rudes coupeurs de têtes » (p. 201) leur ont remis leurs prises, « frénétiquement, leurs épouses [...], avec une férocité bestiale, les font rôtir pour en extraire la cervelle » et la manger (p. 202, voir aussi p. 213, etc.)... Ces descriptions sont rendues encore plus effrayantes par l'emploi d'un «présent ethnologique » indistinct d'un «présent de narration » constant dans l'ouvrage et d'un « présent d'actualité » qui n'y est pas rare non plus. S'il n'est pas exclu qu'il s'agisse d'une recette parmi d'autres en vue de fabriquer un «coup d'éditeur» conforme aux règles du récit à sensation, les ignorances et les complaisances du narrateur et héros de ce bref séjour chez les Asmat révèlent plutôt l'étendue de sa naïveté, ou l'ampleur de celle qu'il attend d'un public entiché d'《arts premiers» aussi bien que d'« expériences vécues ». 
L'ouvrage n'ayant nulle ambition savante, ethnographique ou littéraire, il n'y a pas lieu de discuter de son intérêt sous ces angles-là, non plus que sur le plan du « rêve » ou de « l'escapade onirique » (pp. 262-263) où cherche à le faire valoir (et l'exempter de toute critique) l'auteur inconditionnellement ravi de la préface et de la postface, concluant, entre autres abus de formules et excès de langage, que la parole de Michel Dintrich est « toujours envoûtante ». II n'y a pas lieu davantage (en tout cas ici) d'apprécier, dans ce livre qui se présente sous des auspices poétiques non négligeables (en exergue p. 13, une citation d'Océanie, texte écrit par Breton en 1948), tout ce qui y sépare « le signe et la chose signifiée » dont il se réclame et qui fut énoncée il y a plus de soixante ans d'un tout autre point de vue. Restent certains faits dont la mise en valeur ou l'omission semblent significatives de l'esprit de ce récit d'un voyage entrepris en 1985 et proposé en librairie près d'un quart de siècle plus tard.

À son arrivée à Agats en juillet 1985, le narrateur dut débarquer clandestinement du bateau de la ligne « pionnière » (perintis) de liaison inter-îles de la compagnie de navigation gouvernementale PELNI, sur lequel il était «le seul Blanc» (p. 142) depuis le port d'Ambon, et qui venait de relever des soldats sur la côte mimika. M. Dintrich avait omis de demander l'indispensable permis de voyage (surat jalan), il ignorait apparemment que la région était en ébullition, après les événements de 1981 (emploi de napalm, bombardement d'Enarotali...), le soulèvement raté de Jayapura en 1984, la fuite de milliers de réfugiés en Papouasie Nouvelle-Guinée, et des centaines de civils tués encore au cours du printemps 1985. Heureusement, survinrent des Asmat qui le firent échapper au contrôle de police :

" Athlétiques, debout dans leurs pirogues effilées, ce sont des guerriers olympiens. Leur peau brune souligne le bipane de coquillage blanc qui leur traverse la cloison nasale et leur donne un aspect farouche. » (p. 141)

Quand ils le prirent à bord de leur « frêle embarcation » pour le déposer à Agats, le narrateur se sentit «devenu le héros de ces bandes dessinées [ayant enchanté son] enfance, en particulier celle de Tarzan l'homme-singe ", etc. (p. 142).

C'est aussi en arrivant à Agats qu'il apprit l'existence d'un «musée local» (qu'il ne nomme pas), " composé de plusieurs cases », et se fit remettre par son conservateur (qu'il ne nomme pas davantage) une photocopie (reproduite p. 139) de la carte du pays asmat «établie en 1961 par Michael Rockefeller» avant sa disparition, ainsi qu'une liste « des meilleurs sculpteurs locaux » (reproduite p. 153).

« Je me garde bien, commente M. Dintrich, de lui dire que ses informations vont à l'opposé de ce que je suis venu chercher. En effet, les curios, c'est-à-dire les objets fabriqués pour être vendus aux touristes, n'ont aucun intérêt pour moi : n'étant pas des incarnations d'aïeux, ils n'ont aucune valeur sacrée. Persuadé qu'il existe un art asmat encore vivace [...], je décide de me rendre dans plusieurs villages reculés où je trouverai peut-être mon bonheur. »
Les pages qui suivent narrent comment l'auteur multiplia les visites et les achats auprès des sculpteurs de la liste, dans les villages mêmes qui lui avaient été indiqués sur la carte...

À cette époque, l'Asmat Museum of Culture and Progress, ouvert à Agats en 1973, avait déjà édité plusieurs publications et pouvait se flatter d'avoir contribué à une exposition annoncée dans nombre de pays occidentaux, et notamment à Paris. Présentée au Metropolitan Museum de New York du 11 juin à la mi-septembre 1985, elle s'accompagnait d'un livrecatalogue dû à Tobias Schneebaum, l'un des fondateurs et des plus actifs soutiens du musée d'Agats, avec de remarquables dessins de cet ancien élève de Rufino Tamayo, également visibles au MET à cette occasion. Elle faisait suite aux initiatives d'Adrian A. Gerbrands (à partir de 1960) et surtout de Jac Hoogerbrugge (dès avant 1969) pour encourager les sculpteurs asmat à entretenir leurs traditions, fût-ce en les adaptant aux conditions du marché international de l'art «primitif », voire contemporain. Dès la fin des années 1970, l'arrivée sur le marché occidental de pièces asmat de création récente semait le trouble, suscitant de vives discussions sur "l'authenticité » de tous les objets provenant de cette aire de style, ainsi qu'un intérêt non dénué de suspicion pour les initiatives de l'Asmat Museum.

À Agats même, l'auteur se fit ainsi livrer « un poteau funéraire blanc, ocre et noir, d'environ six mètres de long [...] Le sculpteur auquel je l'avais commandé a travaillé d'arrache-pied pour l'achever avant mon départ [...] Évidemment, le bisj qui m'est destiné n'est qu'un simulacre [...] Il n'empêche que je suis heureux de posséder cette sculpture digne d'un grand musée » (pp. 220-223). Il fit expédier deux caisses d'objets, une première munie d'un "papier officiel [...] sorte de laissez-passer indispensable à la sortie des marchandises hors du pays » (p. 192), et dont il prit livraison en banlieue parisienne quelques mois après (p. 239) et une seconde, chargée à quelques jours d'écart et finalement « saisie par les militaires » indonésiens (p. 244). À Paris, il invita des « amis», un « collectionneur américain », " deux représentants du Musée des arts africains et océaniens ", etc., à contempler ou à se disputer son butin, pour "s'approprier [son] aventure » (p. 242).

Une fois passés ces moments d'effervescence, M. Dintrich dit ne s'être plus intéressé aux Asmat qu'à l'occasion de compositions musicales (en 2003 et 2005) et au hasard d'une brocante parisienne où il dénichait en 2007 «un curios asmat, c'està-dire un objet fabriqué après l'arrivée des missionnaires », qu'il crut devoir acheter (p. 254). L'imperturbable frivolité étalée tout au long de l'ouvrage n'est sans doute pas une attitude isolée, elle pourrait même s'avérer répondre à des attentes de vacanciers enchaînant sur les plages les lectures « d'étonnants voyageurs ». Mais, s'agissant des Asmat et de leur génie plastique, et venant d'un artiste professionnel qui se prétend au fait de l'esthétique occidentale ayant tenu le meilleur compte du « legs des civilisations sauvages", ce livre pousse l'indifférence et l'inintelligence jusqu'au mépris, et il 
ne mérite d'être connu qu'à titre de contre-exemple et de repoussoir.

Gilles BounOuRE

Morphy Howard, 2007. Becoming Art. Exploring Cross-Cultural Categories, Oxford \& New-York, Berg Publishers, 234 p., bibliogr., index, notes, 59 fig. en noir et blanc.

Howard Morphy, directeur de l'École de recherche en humanités à l'Université nationale australienne de Canberra (ANU), tire parti de son expérience de terrain pour remettre en question l'ensemble des catégories d'analyse appliquées à la pratique artistique en contexte ethnique. Son expérience spécifique du Nord de l'Australie, en Terre d'Arnhem, et plus particulièrement auprès des Yolngu, n'est pas le résultat de la rencontre d'un jour, mais d'une longue fréquentation de trente-cinq ans (1973-2008). Mieux, il a été le témoin du changement radical d'appréciation occidentale de la production artistique locale, d'abord considérée comme simple artisanat ethnique (à la limite de l'artefact d'aéroport pour touristes) avant d'être reconnue comme art justiciable du qualificatif de « beaux-arts » ( fine art », p. XI) et comme tel classable dans les grandes collections de niveau mondial. De là, la limpidité du titre de l'ouvrage Becoming Art dédié à ce renversement de perspective (comment ce qui n'était pas vu comme art est, à la génération actuelle, " en train de devenir art»), et du sous-titre stipulant une «exploration de catégories transculturelles ». Autrement dit, l'auteur ne se contente pas d'un compte rendu de terrain, il ambitionne d'en dégager une nouvelle théorie. L'ouvrage analyse le passage du local au global, tout en évitant le piège de la réduction du «global » à l'ethnocentrisme occidental élevé au rang d'un universalisme de jure.

La mise en cause de l'ethnocentrisme de la critique d'art n'est évidemment pas nouvelle. Il y a plus d'un demi-siècle, Claude Lévi-Strauss, par exemple, tenait des propos comparables sur les productions « ethniques » de la Colombie britannique et proférait ce jugement alors anticipateur :

« L'époque n'est pas lointaine, sans doute, où les collections provenant de cette partie du monde quitteront les musées ethnographiques pour prendre place dans les musées des beaux-arts, entre l'Égypte ou la Perse antiques et le Moyen Âge européen. » (1943 : 176)

Une véritable profession de foi, avant la lettre, pour l'intégration des " arts premiers » dans les collections du Louvre, voire un exercice divinatoire pour susciter la création d'un musée du quai Branly privilégiant la dimension esthétique d'objets autrefois simplement ethnographiques!

Ce n'est donc pas tout à fait un hasard si la première figure - sur les cinquante-neuf que recèle l'ouvrage d'Howard Morphy - nous offre justement un coin de plafonds et murs du musée du quai Branly aux couleurs aborigènes, et sur fond de Tour Eiffel en cadrage oblique - cela ne s'invente pas ! L'inspiratrice austra- lienne de ces peintures murales, $\mathbf{M}^{\text {me }}$ Gulumbu Yunupingu, de culture yolngu, apparaît elle-même au premier plan de cette figure, nous permettant de mettre un nom et un visage sur l'œuvre réalisée, ce qui est précisément le propre de toutes les œuvres cotées sur le marché de l'art. Je reviendrai sur l'iconographie de l'ouvrage un peu plus loin. Ce qu'il y a de convaincant dans la démarche de Howard Morphy, c'est que celle-ci va jusqu'au bout de son propos et qu'elle y va même doublement, à la fois en explorant toutes les facettes possibles des catégorisations applicables aux œuvres d'art, et en parcourant jusqu'à son terme l'expérience singulière de la production yolngu de l'Australie du Nord. Pratiques et théories sont dévoilées et analysées d'un même regard.

Les trois premiers chapitres (pp. 27-86) nous installent dans l'histoire de l'art spécifique des Yolngu, de sa période la plus ancienne qui est rapportée par l'auteur au XVIII ${ }^{\mathrm{e}}$ siècle, jusqu'à sa prise en considération dans la catégorie des «beaux-arts» («fine arts») qui se situe au milieu du $\mathrm{Xx}^{\mathrm{e}}$ siècle. Cette première partie est essentiellement descriptive. L'on y apprend que les premières collections d'objets furent constituées par des missionnaires (révérend Chaseling en 1937, p. 48) puis par des anthropologues et des scientifiques (Berndt en 1946, Mountford en 1948, p. 51), avant d'être promues par une exposition spécialement dédiée à l'art aborigène (galerie d'art David Jones à Sydney en 1949, p. 53). Mais le tournant de cette histoire d'art est à dater, selon Morphy, de 1958, année de la décision d'un chirurgien, Stuart Scougall, et d'un directeuradjoint de musée, Tony Tuckson, d'admettre des collections aborigènes à l'Art Gallery of New South Wales (p. 54). Décision historique qui, cette fois-là, a précédé de quelque quarante ans l'initiative du Louvre ou du quai Branly, puisqu'en faisant entrer les œuvres au musée des beaux-arts en lieu et place du musée ethnographique, on s'est mis à requalifier des œuvres « folkloriques » en œuvres d'art à part entière. Pour Morphy, même s'il ne partage pas la distinction sèche entre « artistique » et « ethnographique » (p. 55), le changement d'affectation spatiale fut le signal traduisant un changement de catégorisation dans le classement des œuvres. Cette question est reprise en détail jusqu'à son chapitre 8 , mais elle est transposée dans l'intervalle sur un plan théorique.

La deuxième partie du livre (pp. 87-172) ouvre ainsi la discussion théorique sur les questions de représentation (chap. 5), de style et de signification (chap. 6), ainsi que de discours de légitimation en matière artistique (chap. 7). Sur le plan théorique, la nouveauté de perspective réside dans le positionnement de l'artiste au centre de la fixation de la valeur, et non plus à la périphérie du marché de l'art. Selon l'auteur, le critique d'art n'a plus le monopole du jugement artistique, mais l'artiste local entre dans la dynamique du changement de catégorisation et joue un rôle actif dans la création artistique répondant aux critères internationaux.

Une troisième partie de l'ouvrage, bien que plus succincte (pp. 173-195), peut alors librement s'adonner à appliquer les conclusions tirées de la discussion théorique à la situation de l'artiste local face aux 